\title{
A BIM BASED COLLABORATION PLATFORM FOR DATA TRANSMITTING IN BUILDING DESIGN
}

\author{
Zhuohua Huang ${ }^{1}$, Aizhu Ren ${ }^{2}$
}

\begin{abstract}
BIM (Building Information Modelling) has gradually been accepted and applied by the Chinese engineers. In the meantime, the BIM software which suits the Chinese design process and satisfies the Chinese design codes have not been developed sufficiently. To meet the requirements of BIM based collaboration platform for fluent data transmitting in building design process which suits the Chinese building design practice, this paper presents the solutions on the development of a BIM based collaboration platform. 4-layer architecture is employed to make the function of each part of the collaboration platform clearer; a 3D space for BIM models with version information is created to record the model history and allow engineers to check the version difference; the authorization and certification managing algorithms are designed to ensure an ordered work status on the collaboration platform; the lock-switches and dividing of work sets are developed to solve the object conflict problems; message sending and receiving mechanism are formulated to get fluent communications among the users who are working on the same building project. A protocol of the collaboration platform has been developed on these solutions and shows the study results.
\end{abstract}

Keywords: BIM, Collaboration platform, Building Design, System architecture.

\section{INTRODUCTION}

BIM (Building Information Modelling) has gradually been accepted and applied by the Chinese engineers along with the rapid development of BIM technology, while there are still some problems for the employment of BIM in the Chinese engineering practice. For instance, the BIM application software which suits the Chinese design process and satisfies the Chinese design codes have not been developed sufficiently, the localization of the existing BIM software is not adequate to meet the requirements of Chinese engineering practice, the BIM data transmitted in different software are not fluently, which reduced significantly the design efficiency. Therefore, it is urgent to develop a BIM based collaboration platform for fluently data transmitting in building design which suits the Chinese design process, satisfies the Chinese design codes.

This paper presents the new methods and algorithms employed in the development of a BIM based collaboration platform for the new BIM software. The BIM based collaboration platform can provide well communication means so that the architectures, the structural engineers and HVAC Engineers may work on their different BIM models and communicate their ideas and BIM data via the collaboration platform effectively.

1 Engineer, Institute of Building Engineering Software, CABR Tech Co., LTD, Beijing, China. huangzhuohua@cabrtech.com

2 Professor, Department of Civil Engineering, Tsinghua University, Beijing, China. raz-dci@tsinghua.edu.cn 
A. JalyZada studied the BIM based Versioning for collaborative design, and proposed several new information modelling entities as an extension to the current IFC standard (JalyZada et al. 2014). Jun Wang developed a framework to explain the design collaboration process by incorporating the BIM technology in addressing the design collaboration for a complex building (Wang et al. 2014). C. Merschbrock analysed a building project comprising a network of organizations interrelated by their information systems, to gain a better understanding of collaborative design based on BIM (Merschbrock 2012).

In addition to these achievements, there are still some technical problems should be solved in the development of a BIM software for the building design practice (Tomo 2011). For instance, a BIM based collaboration platform should be developed so that the professional individuals can work on the BIM models without object conflict, the building project data can be transmitted fluently when it is calling remotely, etc. Therefore, this paper presents the authors' solutions on the development of BIM based collaboration platform.

\section{ANALYSIS OF SYSTEM REQUIREMENTS}

In the most building design institutes in China, the architects, structural engineers, HVAC engineers, water supply and drainage engineers, electrical and electronical engineers are organized as a group. The study on the existing building design institutes in China shows, there are three types of BIM applications:

-The collaboration group may be small and have no full-time BIM manager. Web Server, BIM Sever and Database Server are laid in one sever.

-There may be a full time or part time BIM manager. Web Server, BIM Sever and Database Server are laid separately in a different sever or sever group. In addition, there are a publication sever and a file Sever.

-There may be full time BIM managers. A local buffer sever is laid for the clients, a load balance sever is laid amongst the several groups of clients. In addition, the buffer severs, the high capability cloud computing server and cloud storage are also laid to meet the business requirements for those large, international design institutes.

Based on the testing of networks and severs, the system configuration requirements are designed as follows:

(1) Network environment:

1) LAN (local area network). In a normal condition, the connection between the database server and the application server should be at least $100 \mathrm{M}$ bps. For the large design institutes, the connection between the database server and the application server should be at least $1000 \mathrm{M}$ bps.

The bandwidth from client to sever should be more than $10 \mathrm{M}$ bps; from server to sever should be more than $100 \mathrm{M}$ bps. The wire delay should not be higher than $10 \mathrm{~ms}$, the packet loss rate should not be higher than $0.1 \%$.

2) WAN (Wide area network). The bandwidth from the client to sever should be at least $1 \mathrm{M}$ bps ; the bandwidth from sever to sever should be more than $10 \mathrm{M}$ bps. The wire delay should not be higher than $100 \mathrm{~ms}$, the packet loss rate should not be higher than $1 \%$.

3) Firewall. Firewalls should be installed in a design institute for the data safety. 
(2) System environment

The system environment requirements are as shown in Table 1.

Table 1: System Environment requirements

\begin{tabular}{|c|c|c|}
\hline $\begin{array}{l}\text { Integration } \\
\text { Server }\end{array}$ & \multicolumn{2}{|c|}{$\begin{array}{l}\text { Hard Disk: 2TB enterprise-level (RAID 1); } \\
\text { Network Card: 1Gigabytes Ethernet card. }\end{array}$} \\
\hline $\begin{array}{l}\text { Database } \\
\text { Server }\end{array}$ & \multicolumn{2}{|c|}{$\begin{array}{l}\text { Hard Disk: 2TB enterprise-level (RAID 5); } \\
\text { Network Card: 1Gigabytes Ethernet card. }\end{array}$} \\
\hline $\begin{array}{l}\text { Application } \\
\text { Server }\end{array}$ & \multicolumn{2}{|c|}{$\begin{array}{c}\text { Hard Disk: 1TB; } \\
\text { Network Card: 1Gigabytes Ethernet card. }\end{array}$} \\
\hline WEB Server & $\begin{array}{l}\text { CPU: Core } 2 \text { Quad or higher; } \\
\text { Hard Disk: } 2 \mathrm{~TB} \\
\text { Network Card: 100M bps }\end{array}$ & $\begin{array}{l}\text { Memory: 4GB; } \\
\text { et card. }\end{array}$ \\
\hline
\end{tabular}

Based on the analysis of the different size and level of design institutes, the collaboration platform function requirements can be formulated (Huang et al. 2013). The users can access the building project through the traditional PC client program, web pages or mobile devices. Though there are variable design institutes, the basic system functions are almost the same. The difference amongst them is the user amount, the data volume, the institute management systems, etc. The major functions for a collaboration platform are: synchronous and asynchronous managing, data storing and transmitting, authority managing, message sending and model version managing, etc.

Figure 1 shows a hardware configuration for a small design institute.

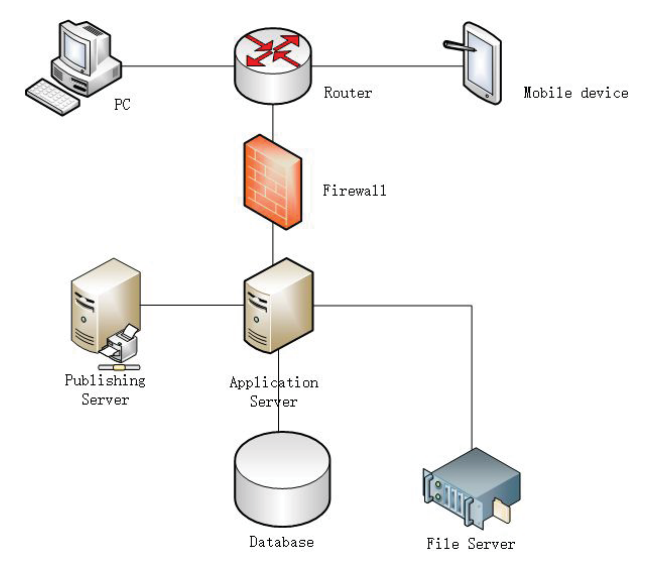

Figure 1: A hardware configuration for a small design institute. 


\section{SYSTEM ARCHITECTURE}

The collaboration platform should face to heterogeneous networks, different user requirements from different professions, complicated design path, multiple project groups, etc. Multi-Layer Architecture is a suitable solution to solve this problem. In a Multi-Layer Architecture, the professional logic, the data operation and the user interaction are separated; the codes of a module are relatively independent; different profession can be distributed on the different computer; the data layer is isolated with the profession layer. This feature makes the function of each part of the platform clearer, so that the software development efficiency is improved, and the error rate decreased significantly.

Based on the analysis of system requirements and the consideration of data security, the authors employ 4-layer architecture for the design of collaboration platform as shown in Figure 2. They are interface layer, profession logic layer, model layer and data maintaining layer.

The interface layer displays the system prompts to the users such as icons, dialogue box, data, messages and tables; echoes the user input texts, data, graphical elements and selection items; control the model view; manage the user selection and achieves the communication between the system and the users.

The profession logic layer is corresponding to the applications, which explains and maintains the design profession logic; achieves the synchronization or asynchronous collaboration work; completes the user appointing operation; manages the user requests; return the system managing results to the user requests.

The model layer defines the data structure and the relationship between two data structure, organizes the data provided by the data maintain layer as a professional model for the profession logic layer, holds the data convention and data analysis logic .

The data maintaining layer organizes and stores the data in object oriented structure for a long term. ORM (Object Relational Mapping) technology is employed in this layer since ORM provides functionality to perform complete CRUD operations and encourages object-oriented querying, so that the direct database operating can be avoided. The portability, extendibility and maintainability of the collaboration platform are therefore ensured. The existing of the data maintaining layer ensures the safety and secrecy of the data shared by the project group members, and the correctness of the data accessing.

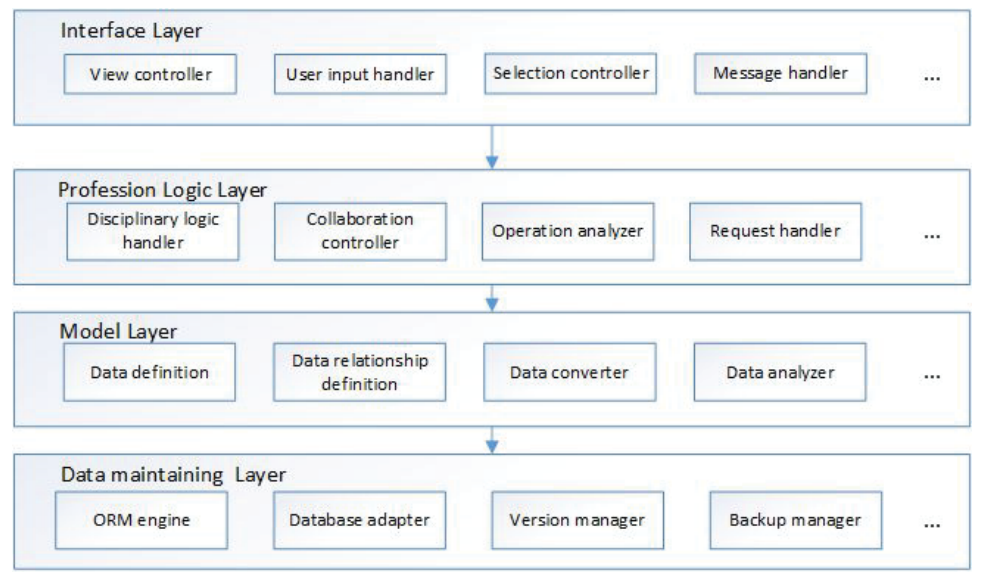

Figure 2: The system architecture 
To meet the user requirements from different size of design institutes, SOA (ServiceOriented Architecture) is employed. SOA is a component model which links the different function unit of an application and the agreement by the well specified interface between the services. The interface is specified by a neutral way, which is independent of the hardware, the operation system and programming language.

By employing 4-layer architecture and SOA technology, the safety and secrecy of remote accessing and transmitting of the data can be ensured easily. The functions for remote calling via network includes the communication agency for the client-side and the sever-side which execute the distributed deployments, the conversation management, the message sending and response, and the reflection calling, etc.

\section{A 3D SPACE FOR BIM MODELS WITH VERSION INFORMATION}

It is not suitable to store the whole BIM model of a building project in a single data set while multiple users are working on the same BIM model considering the capacity of the current available hardware and software, the user operating convenience, and the data transmission speed. Along with the design process of a building project, the BIM model will be modified frequently. It is necessary to carefully specify the structure, the version, and the relations between the individual professions of a BIM model. Fig. 3 displays the specification of BIM model at a specific design stage for a building project by the authors.

Fig. 3 displays BIM models in a specific design stage which forms a 2D plane. The core model is the foundation of the collaboration design, which is built by the BIM manager in accordance with the responsible person's instruction of the project, and is allowed only by the BIM manager to be modified in a case requested by a designer and is approved by the responsible person of the project (Ren 1987). The basic information of a building such as axis lines, walls, columns and floors are presented in the core model, based on which, the architects, structural engineers, HVAC engineers and power \& electronic engineers can build their architectural model, structural model, HVAC model and power \& electronic model, which can be accessed by other professional engineers. In turn, the architects, structural engineers, HVAC engineers and power \& electronic engineers build their private profession models which can be accessed only by themselves. The information which is not necessary is presented in the private profession models.

The access to the core model or to the architectural model, structural model, HVAC model, or power \& electronic model, is managed by a uniform interface. A member of the collaboration design group is assigned specific authorization to access a model via the uniform interface. If a designer finds that a component of his model is passed through by a component of another professional model, he may send a request for modifying the other professional model via the uniform interface, the other professional engineer may reply to accept or not accept the request via the uniform interface.

The design process of a building project normally has 3 stages in China: preliminary design, technical design and construction drawing design. Every design step between two design stages is specified by date. The BIM models in the design process form a 3D space. The version information of a BIM model indicates the date information (on which the BIM model was built) in the vertical axis. Modifications happen frequently along with the design process. By accessing the version data, the user can modify the BIM model at any design step. The design process can also be reviewed via the version date. 


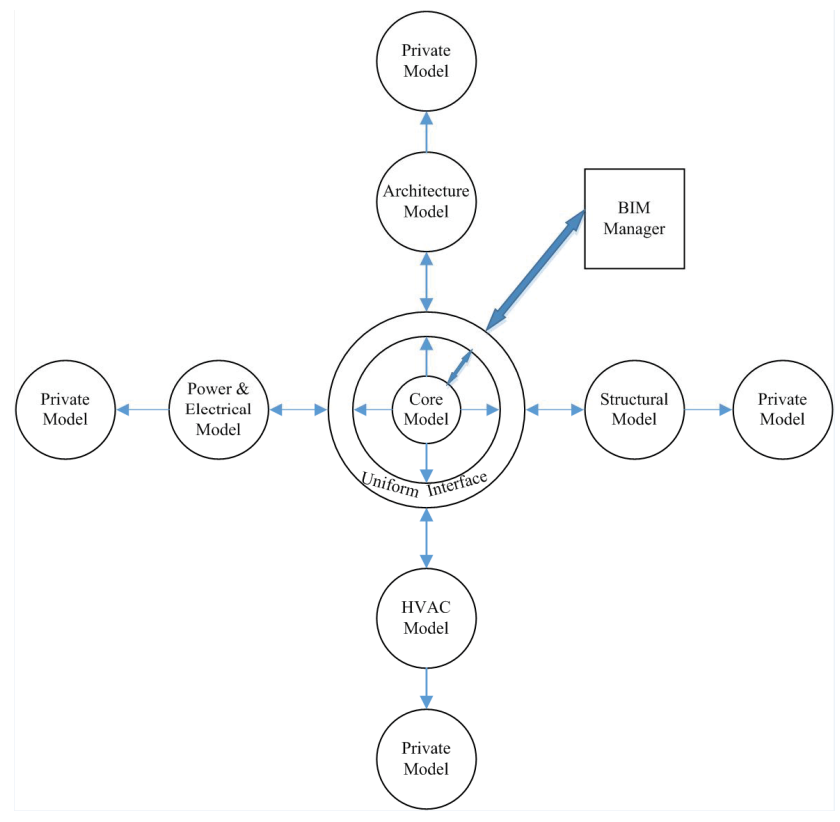

Figure 3: Specification of BIM models

The class inheritance diagram is displayed in Figure 4. Take a wall as an example, the shared data amongst professions are defined as the base classes. When they are to be interchanged amongst different professions, the base classes can be transformed to the other system completely (Remond et al. 2012).

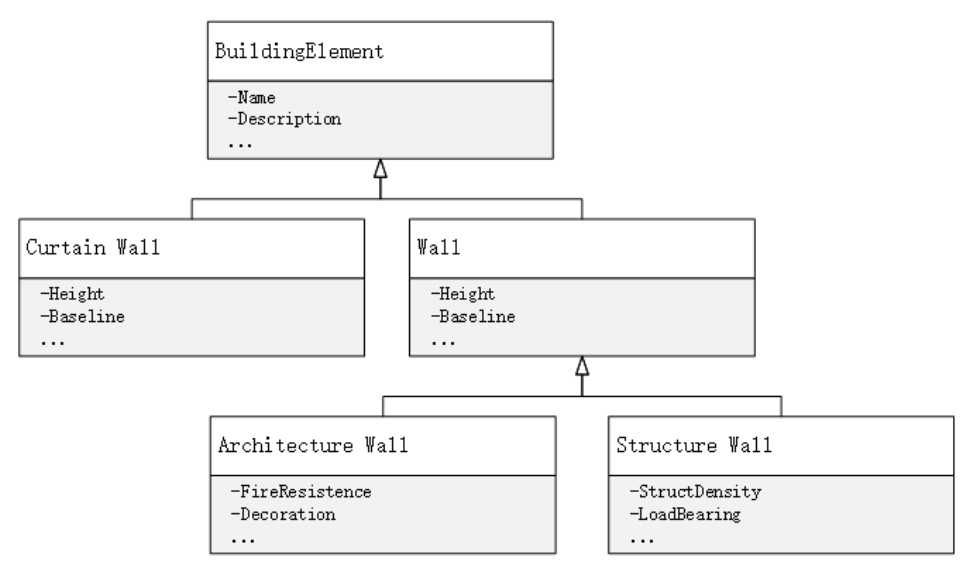

Figure 4: The class inheritance diagram

\section{AUTHORIZATION AND CERTIFICATION}

The collaboration platform is a distributed multi-user designing system that allows different users at different places to work together on the same building project. It allows the users to access the building project through the traditional PC client program, web pages or mobile devices, the users can browse the components that the other users are working on and notify the others if there is anything wrong. The data server stores all project data and all user information; it gives data access to the clients and ensures that only the user with the right privilege can get the project data. 
To ensure that all the data are transmitted in a correct and safe way via Internet or Intranet, a role manager is developed in the collaboration platform. The role manager has the functions to confirm the person's identity before the user accesses the data in the client, to sign his name in sending his messages, and to encrypt the messages before they are sending, so that the homogeneity and the confidentiality of the messages can be ensured (Scheer et al. 2012). In spite of this, a reliable conversation mechanism is developed to ensure the order and non-repeating of the data transmitting.

The role manager is designed as follows:

(1) All the roles in a project should be assigned by the BIM manager in accordance with the project responsible person's instruction. The BIM manager has the authority to set up the authority for each role.

(2) A user may have one or more than one roles. A role may correspond to a specific working area. Every user's operation is verified via the authority information in the function table.

Before the design group starts working on a project, the BIM manager should initialize the system, all the function tables of the users and the roles, the groups are then initialized, and the correlation between a user and a role, between a role and the function are in turn initialized. As soon as a user logs on, all the function menus and function tables of the user's roles are loaded. As soon as a user asks an operation, the system will verify the authority according to the user role and its function-authority relation.

\section{SENDING AND RECEIVING OF MESSAGES}

To provide fluent communications among the designers during a collaboration work process, communication support tools are developed via a message and communication module, so that the users' messages can correctly reach the destination in time.

A message is generated by a designer via immediate communication interface, or triggered by the system or a project event. As soon as the message is saved on the server, the message distribute program will push the newly entering message to the client-side.

A message may be texts, graphs or other attachments, which is corresponding to the progress of a project. As a part of a project data, the messages are stored at the sever-side and can be trace back to later operations.

The system is developed such that a message will reach the designations immediately. The messages on the sever can be saw at any time. A filter is developed to screening the contents for the users. The filter has the priority to filter the contents before a message is pushing or getting.

A project progress monitor is developed, so that the designers can be reminded on his duty to finish in time. By setting the planned start date, the planned end date, the real start date, the real end date, expect date to remind the users, and expect person to be reminded, the system will pop a box.

\section{AN EXAMPLE FOR APPLICATION OF COLLABORATION PLATFORM}

Based on the above solutions, a protocol of the collaboration platform with the following features has been developed:

(1) The number of users is not lager than 500. The number of on-line users is not lager than 150. The number of concurrency is not lager than 50 . 
(2) The response time to a single log on operation is 0.1 second for Ethernet, 1 second for Internet. The response time to an upload/download operation for $10 \mathrm{M}$ data is 2 seconds for Ethernet, 15 seconds for Internet.

(3) The important data which are to be transmitted is encrypted, and the network accessing will be verified in advance. The countermeasure to interrupt, intercept, modify and forge attacks is provided. The protocol can reply DDOS attacks from 10 terminals.

(4) Data back-up and recovery functions are provided. The recovery time of $10 \mathrm{~GB}$ data is no longer than 3 hours.

(5) All the operations are stored in an operation daily record. The illegal or destructive operation can be found from the daily record as soon as a safety problem occurs.

Fig. 5 displays multiple users among different profession groups are working on the same building project.
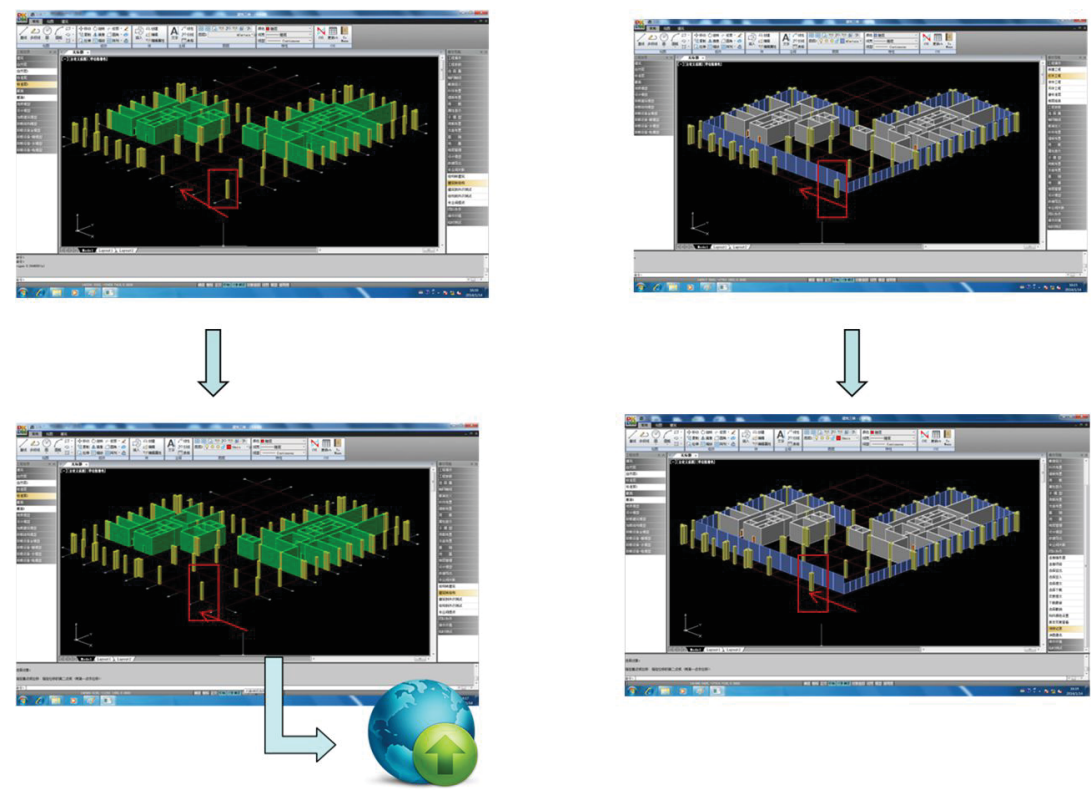

Figure 5: Multiple users are working on the same building project

There are two ways of the collaborative platforms applied in China:

(1) By linking of the Autodesk Revit and AutoCAD associated folders and the collaborative design cloud to realize collaborative design. Some Chinese design institutes employs Revit and AutoCAD to build 3D architectural models and interchange project messages.

(2) By developing the conversion tools to transform models from one software such as Revit to another software such as SAP2000.

Compare to the two above ways, the collaborative platform presented in this paper has the centre data base which has rapid access speed.

\section{CONCLUSIONS}

This paper studied the solutions on the development of a BIM based collaboration platform. The study results can be concluded as follows: 
(1) 4-layer architecture makes the function of each part of the collaboration platform clearer, so that the software development efficiency is improved, and the wrong or illegal access on data base decreased significantly.

(2) The creation of a 3D space for BIM models with version information decreases the data transmitting volume and accessing frequency significantly.

(3) The authorization and certification managing algorithms ensures an ordered work status for all the users on the collaboration platform.

(4) Development of message sending and receive mechanism makes the communications among the users who are working on a building project at the same time fluently.

Because of the limitation of the paper pages, some technical details and other solutions are not presented in this paper. The authors will present them in the other papers.

\section{ACKNOWLEDGMENTS}

The authors are grateful for the financial support received from the National Key Technology R\&D Program (No. 2015BAK14B02).

\section{REFERENCES}

JalyZada, A. et al. (2014). Building Information Modelling (BIM)-Versioning for collaborative design. Computing in civil and building engineering. @ ASCE 2014 p512.

Wang, J. et al. (2014). Cooperative Design, Visualization, and Engineering. 11th International Conference, CDVE 2014. Seattle, WA, USA,14-17.

Merschbrock, C. (2012). Collaboration in multi-actor BIM design: A configuration analysis view. 9th European Conference on Product and Process Modelling. Reykjavik, ICELAND. JUL 25-27, 2012

Tomo, C. (2011). A review and outlook for a 'Building Information Model1 (BIM): A multistandpoint framework for technological development. Advanced Engineering Informatics, 2011(02): 224-244.

Huang, Z., Liu, Y. and Ma E. (2013). Mobile application development in a collaborative AEC designing system. Proceedings of 30th International Conference on Application of IT in the Construction Industry, 9-12, October 2013. Beijing, China.

Ren, A. and Pahl, P.J. (1987). RENSYS-Dialogue System for Structural Analysis User Manual. IAB-Bericht 6. Berlin 1987. ISBN 3798311781

Remond, A. et al. (2012). Exploring how information exchange can be enhanced through cloud BIM. Automation in Construction, 2012,24: 175-183.

Scheer, S. et al. (2012). Building design teamwork supported by a collaborative CAD modelling process analysis. Proceeding of the 13th International Conference on Computing in Civil and Building Engineering. June 2012, Nottingham, UK. 